\title{
Prävention und Bekämpfung von häuslicher Gewalt
}

\section{Sibil Tschudin}

PD Dr. med., Leitende Ärztin Abteilung Gyn. Sozialmedizin/Psychosomatik, Frauenklinik Universitätsspital Basel

\section{Häusliche Gewalt - ein Thema in der Medizin?}

Die Frage, ob häusliche Gewalt ein Thema in der Medizin ist, würden alle Mitarbeiterinnen der von mir geleiteten Abteilung für gynäkologische Sozialmedizin und Psychosomatik und darüber hinaus wohl auch alle anderen in der Frauenklinik des Universitätsspitals Basel tätigen Ärztinnen und Ärzte, Pflegenden und Hebammen mit Ja beantworten. Wir sind sensibilisiert durch die Berichte über sexuelle und körperliche Traumatisierungen durch Partner oder fremde Täter an den Morgenrapporten und fühlen uns immer wieder bestätigt, wenn sich Verdachtsmomente - gerade bei Schwangeren - auf Nachfrage hin als richtig erweisen. Von häuslicher Gewalt betroffene Patientinnen werden von meinem Team (mit-)betreut, wobei grosser Wert auf die Zusammenarbeit mit dem Sozialdienst und eine gute Vernetzung mit den kantonalen Institutionen und Behörden, die mit der Opferhilfe betraut sind, gelegt wird. Wenn es naheliegend ist, dass Frauenkliniken mit dem Thema konfrontiert sind, wie sieht es dann mit

\begin{tabular}{l} 
Tabelle 1: Risikofaktoren für häusliche Gewalt. \\
\hline Strukturell \\
\hline Geschlechtsungleichheit \\
\hline Politische Instabilität \\
\hline Kulturelle Akzeptanz von Gewalt \\
\hline Institutionell \\
\hline Tiefer sozioökonomischer Status \\
\hline Arbeitslosigkeit \\
\hline Armut \\
\hline Isolation \\
\hline Interpersonell \\
\hline Familienkonflikte \\
\hline Mangelnde Kontrolle über Versorgung der Familie \\
\hline Alkohol-/Drogenkonsum \\
\hline Individuell \\
\hline Gewalterfahrung in der Lebensgeschichte \\
\hline Ablehnung durch die Familie \\
\hline (Unerwünschte) Schwangerschaft \\
\hline Psychische Erkrankungen \\
\hline
\end{tabular}

den anderen Fachdisziplinen aus? Zahlreiche Erhebungen haben ergeben, dass die Prävalenz häuslicher Gewalt hoch ist und dass sich von Gewalt betroffene Frauen am häufigsten an Institutionen des Gesundheitswesens wenden $[1-3]^{*}$. Somit sollten sich nicht nur Gynäkolog(inn)en, sondern alle Ärztinnen und Ärzte, die Frauen jeden Alters betreuen, bewusst sein, dass gut 10\% ihrer Patientinnen Erfahrungen mit häuslicher Gewalt haben dürften. Häusliche Gewalt tritt in allen sozialen Schichten unabhängig von Bildungsstand, religiöser und ethnischer Zugehörigkeit, Einkommen und Alter auf. Gleichwohl gibt es gewisse Risikofaktoren, wie Tabelle 1 zeigt [4]. Zudem erkennen Frauen, die Gewalt in ihrer Herkunftsfamilie erlebt haben, die Gewalt, die sie in ihrer Beziehung erfahren, oft lange nicht als solche.

\section{Häusliche Gewalt - Welchen Beitrag können Ärztinnen und Ärzte zu Prävention und Bekämpfung leisten?}

\section{Betreuung von Opfern häuslicher Gewalt}

Zwar steigt die Zahl der Opfer, die nach häuslicher Gewalt medizinische Hilfe suchen und eine Anzeige erstatten; aber noch immer scheuen viele Frauen diesen Schritt. Einerseits besteht nicht selten - auch nach wiederholten Aggressionen - die Hoffnung, dass der Partner seine Versprechungen, sich zu bessern, erfüllt. Andererseits hindern häufig Ängste vor Repressionen seitens des Täters die betroffene Frau daran, einer medizinischen Fachperson, der Beraterin in einer Anlaufstelle für Gewaltopfer oder gar der Polizei von ihrer Gewalterfahrung zu berichten Gleichzeitig benötigen diese Frauen dringend Unterstützung. Entsprechend ist es wichtig, dass alle Medizinalpersonen, die Patientinnen betreuen, vertraut sind mit dieser Problematik. Sie sollten sensibilisiert sein auf das Erkennen von Zeichen, die auf erfahrene Gewalt hinweisen, wissen, was beim Umgang mit Gewaltopfern speziell beachtet werden sollte und über die nötige Beratungskompetenz verfügen $[4,5]$. Für eine adäquate Betreuung braucht es allerdings 
kein weitreichendes Expertenwissen, wichtiger ist die Kenntnis von Anlaufstellen und Betreuungsnetzen für Gewaltopfer. Die Art der Unterstützung, welche die Patientin braucht, richtet sich nach der Situation, in der sie sich befindet. Eher selten ist dies die Akutsituation unmittelbar nach einer körperlichen und/oder sexuellen Traumatisierung, häufiger handelt es sich um Situationen, die Gewalt vermuten lassen, oder in denen Patientinnen von sich aus über erfahrene häusliche Gewalt berichten. Eine professionelle ärztliche Betreuung von Gewaltopfer zeichnet sich dadurch aus, dass Ärzte Offenheit gegenüber dem Thema signalisieren, Unterstützung anbieten, aber nicht bevormundend sind und Respekt gegenüber der Betroffenen und dem von ihr gegangenen Lösungsweg bekunden. Auch um der eigenen Überforderung entgegenzuwirken, ist es wichtig, dabei Grenzen wahrzunehmen und auch zu setzen.

Die ärztliche Reaktion auf eine gewaltbetroffene Frau, wenn sie über ihre Situation spricht, ist von besonderer Bedeutung. Die Art und Weise, wie ihr begegnet wird, stellt die Weichen für die weitere Verarbeitung der traumatischen Erfahrungen und dafür, inwieweit sie weitere Hilfeangebote in Anspruch nehmen kann.

\section{Screening auf häusliche Gewalt}

Aufgrund der Tatsache, dass häusliche Gewalt und daraus resultierendes Leiden häufig lange nicht erkannt wird, stellt sich die Frage, ob es nicht sinnvoll und angezeigt wäre, dass Ärztinnen und Ärzte im Anamnesegespräch routinemässig danach fragen. Das Ziel eines solchen Screenings wäre es, den Frauen einerseits zu vermitteln, dass ihre Ärztin / ihr Arzt auch diesem Thema gegenüber offen ist, und andererseits den Frauen die Möglichkeit zu geben, über ihre Gewalterfahrungen zu sprechen. Die Umfrage in der Frauenklinik Maternité Inselhof zeigte, dass neun von zehn Frauen einem Screening gegenüber positiv eingestellt waren [2]. Eine Frau legte allerdings grossen Wert auf die Art und Weise, wie gefragt wird.

Wird die Frage nach häuslicher Gewalt mit einer kurzen Erklärung eingeleitet, z.B. mit einem Hinweis auf die Häufigkeit des Problems und darauf, dass die Frage routinemässig allen Frauen gestellt wird, so wird sie in der Regel gut aufgenommen [4]. Nicht betroffene Frauen verneinen erleichtert, betroffene können sich dadurch ein erstes Mal ermutigt fühlen, sich der Ärztin / dem Arzt anzuvertrauen, oder sie haben zumindest erfahren, dass es da eine Ansprechperson mit Verständnis gäbe, an die sie sich zu einem späteren Zeitpunkt wenden könnten. Selbstverständlich muss bei der routinemässigen Befragung darauf geachtet werden, dass die Frau nicht in der Gegenwart von Familienangehörigen gefragt wird oder diese zum Dolmetschen beigezogen werden.

\section{Häusliche Gewalt - ein Thema in der Aus-, Weiter- und Fortbildung?}

Häusliche Gewalt ist also ein Thema in der Medizin und Ärztinnen und Ärzte können auch einen sinnvollen Beitrag bei der Erkennung und Betreuung von gewaltbetroffenen Frauen leisten. Aber sind sie auch adäquat darauf vorbereitet?

Häusliche Gewalt ist nur ganz am Rande oder gar nicht Thema während des Medizinstudiums; im Stoffkatalog der Medizinischen Fakultät Basel findet sich jedenfalls kein entsprechendes Stichwort.

Was die Weiterbildung anbelangt, so enthält einzig der Lernzielkatalog für Hausarztmedizin das Stichwort «Häusliche Gewalt». In der Gynäkologie/Geburtshilfe ist das Thema häusliche und sexuelle Gewalt für die deutschsprachigen Kandidat(inn)en seit 2010 offizieller Bestandteil des Kursprogramms der obligatorischen ins Curriculum integrierten Weiterbildung in psychosomatischer Kompetenz und umfasst eine interaktive Unterrichtseinheit von 75 Minuten.

Eine Arbeitsgruppe der Schweizerischen Gesellschaft für Gynäkologie und Geburtshilfe (SGGG) hat 2008 einen Leitfaden zur Verbesserung der Betreuung betroffener Frauen für ihre Mitglieder verfasst [4]. Er wurde 2009 am Jahreskongress der SGGG im Rahmen eines Hauptthemas vorgestellt und ist über die Homepage der Fachgesellschaft für alle zugänglich. Am Universitätsspital Basel wurde 2004 eine Studie durchgeführt, bei der Mitarbeitende verschiedener Berufsgruppen zur Konfrontation mit häuslicher Gewalt im Berufsalltag befragt wurden [6]. Es zeigte sich, dass das Thema als Problem erkannt war, aber Wissensdefizite und Handlungsunsicherheiten bestanden. Allerdings liess der Besuch der daraufhin entwickelten Fortbildungsangebote rasch wieder nach, was deutlich macht, dass das belastende Thema immer wieder gerne an den Rand gedrängt wird.

Die nationale Konferenz zur häuslichen Gewalt als Thema der öffentlichen Gesundheit, die im November 2014 in Bern stattfand, hat die Bedeutung und Tragweite des Problems einmal mehr deutlich gemacht. Nun gilt es, die vielen guten Ansätze, die schweizweit bestehen, zu bündeln und auszubauen. Eine verstärkte Implementierung des Themas in die Aus-, Weiter- und Fortbildung wäre eine wichtige Voraussetzung dafür, dass wir Ärztinnen und Ärzte unseren Beitrag zur Prävention und Bekämpfung häuslicher Gewalt optimal leisten können. 


\section{Références}

1 Facts and Figures: Ending Violence against Women. www.unwomen.org/en/what-we-do/ending-violence-againstwomen/facts-and-figures

2 Gloor D, Meier H. Frauen, Gesundheit und Gewalt im sozialen Nahraum. Repräsentativbefragung bei Patientinnen der Maternité Inselhof Triemli, Klinik für Geburtshilfe und Gynäkologie. Bern: Edition Soziothek; 2004.

3 Spitzberg BH. An analysis of empirical estimates of sexual aggression victimization and perpetration. Violence and victims. 1999; 14(3):241-60.
4 Bass B, Ghavami-Dicker S, Pók Lundquist J, Schnarwyler B, Tschudin S, von Castelberg B, et al. Leitfaden Häusliche Gewalt - Verbesserung der Betreuung betroffener Frauen. 2008. http://sggg.ch/de/content/leitfaden-häusliche-gewalt

5 Tschudin S. [Immediate care for women after sexual and physical assault]. Die Erstbetreuung von Frauen nach sexueller und körperlicher Gewalt. Therapeutische Umschau. 2005; $62(4): 223-9$

6 Gloor D, Meier H, Häusliche Gewalt bei Patientinnen und Patienten. Eine sozialwissenschaftliche Studie am Universitätsspital Basel. Schriftenreihe des Justizdepartements. Band 8; Basel; 2005 